\title{
Cushing's disease - medical chameleon. Case report of the patient with cyclic, ACTH-dependent Cushing's syndrome due to atypical pituitary macroadenoma

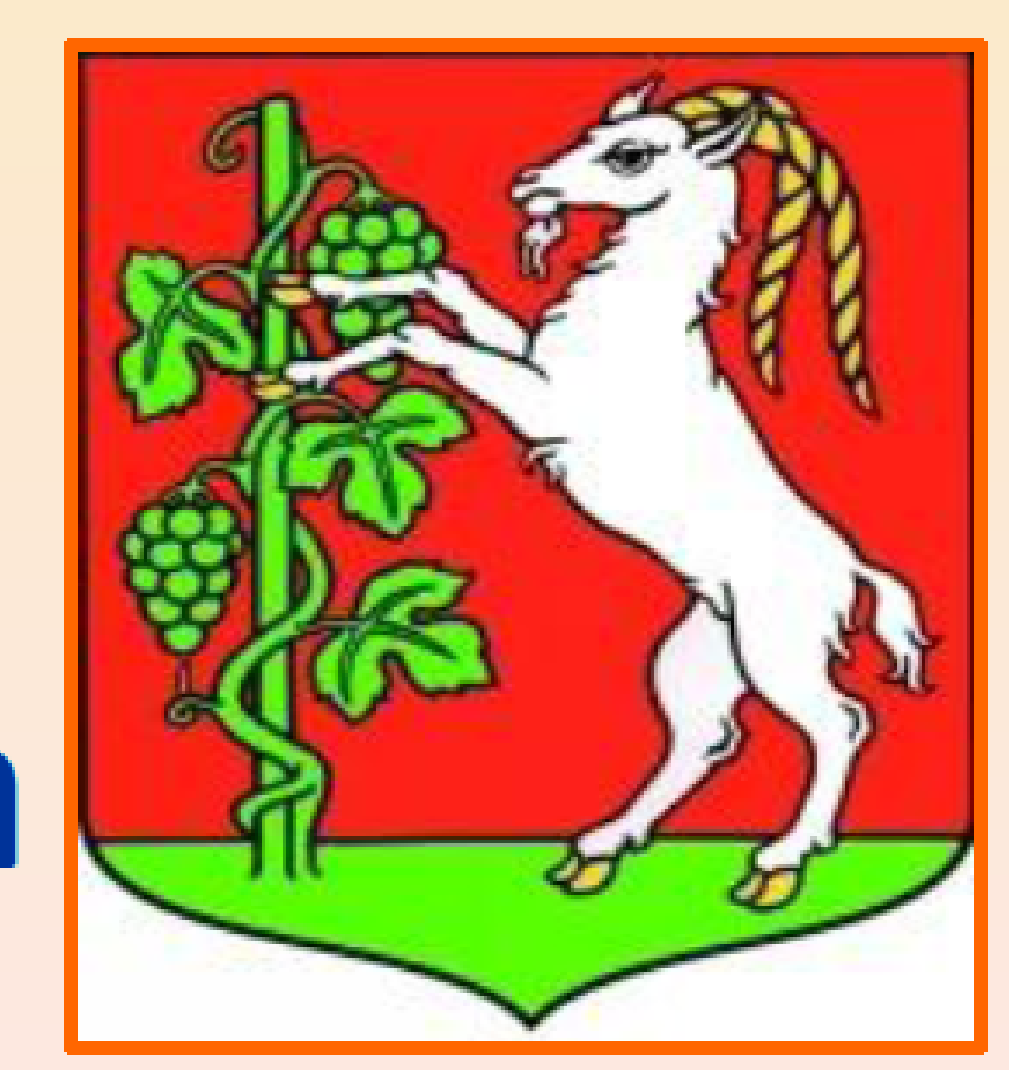

\author{
${ }^{1}$ Joanna Malicka, ${ }^{2}$ Marta Dudzińska, ${ }^{1}$ Maria Kurowska, ${ }^{1}$ Jerzy S. Tarach
}

\author{
${ }^{1}$ Department of Endocrinology, Medical University, Lublin, Poland \\ ${ }^{2}$ Chair of Internal Medicine and Department of Internal Medicine in Nursing, Medical \\ University, Lublin, Poland
}

Introduction. ACTH-secreting macroadenomas account for about $4-10 \%$ of Cushing's disease and are often resistant to surgical treatment and radiotherapy.

The aim of the study was to present diagnostic and therapeutic difficulties in a case of cyclic recurrent ACTH-dependent Cushing's syndrome due to atypical pituitary adenoma.

Case report. In 08.2007 50-year-old man with visceral obesity was referred to hospital because of poor control of diabetes and high blood pressure. On the basis of clinical symptoms and severe hypokaliemia hypercortisolemia was suspected and laboratory test were performed. They confirmed ACTH-dependent Cushing's syndrome (tab.1). Further investigations did not show any pathology of the pituitary. Ectopic source of ACTH excess was not found either. Between 08.2007 and 03.2008 the patient received inhibitors of steroidogenesis (ketoconazole, aminoglutetimid). The loose of weight $(30 \mathrm{~kg})$, normalization of cortisol and ACTH levels and significant improvement of patient's well-being were observed. The treatment has been withdrawn.

\begin{tabular}{|c|c|c|c|c|c|c|}
\hline Date & $\begin{array}{c}\text { Weight } \\
{[\mathrm{kg}] /} \\
\mathrm{BMI} \\
{\left[\mathrm{kg} / \mathrm{m}^{2}\right]}\end{array}$ & $\begin{array}{c}\text { Cortisol } \\
\text { plasma } \\
8.00 \mathrm{am} \\
\text { [ug/dl] } \\
\text { (range 4,3- } \\
22,4 \text { ) }\end{array}$ & $\begin{array}{c}\text { ACTH } \\
\text { plasma } \\
{[\mathrm{pg} / \mathrm{ml}]} \\
\left({ }^{1} \text { range }\right. \\
4,7-48,6, \\
{ }^{2} \text { range } 7,2- \\
63)\end{array}$ & $\begin{array}{c}\text { 24-hour } \\
\text { urinary free } \\
\text { cortisol } \\
\text { [ug/24h] } \\
\text { (range 32- } \\
243 \text { ) }\end{array}$ & Treatment & Pituitary MRI \\
\hline $\begin{array}{l}\text { 08.2007- } \\
\text { diagnosis }\end{array}$ & $112 / 35$ & 46,6 & $176^{1}$ & 15870 & $\begin{array}{c}\text { Ketoconazole 600- } \\
800 \mathrm{mg}\end{array}$ & normal \\
\hline 11.2007 & $102 / 32$ & 8,7 & $53^{1}$ & $67,6-180,0$ & $\begin{array}{l}\text { Aminoglutetimid } \\
750 \mathrm{mg}\end{array}$ & normal \\
\hline 09.2008 & $79 / 24,6$ & 10,5 & $31,3^{1}$ & 304 & without treatment & \\
\hline 10.2009 & $78 / 24,3$ & 13,3 & $57,9^{1}$ & 260 & without treatment & \\
\hline 08.2010 & $77,7 / 24$ & 10,6 & $81,7^{1}$ & 330 & without treatment & normal \\
\hline 11.2011 & $82 / 25,5$ & 16,7 & $109,9^{2}$ & 696 & without treatment & normal \\
\hline 10.2012 & $82 / 25,5$ & 27,2 & $115^{2}$ & 1285 & without treatment & \\
\hline 05.2013 & $88,3 / 27$ & 73,4 & $217,5^{2}$ & 10336 & $\begin{array}{l}\text { Ketoconazole } \\
400 \mathrm{mg}\end{array}$ & $\begin{array}{c}\text { pituitary } \\
\text { macroadenoma } \\
15 \times 13 \times 15 \mathrm{~mm} \\
\text { (fig. } 1 \text { and } 2 \text { ) }\end{array}$ \\
\hline 09.2013 & $83 / 25,9$ & 19,6 & $75,6^{2}$ & 673 & $\begin{array}{l}\text { transsphenoidal } \\
\text { adenomectomy }\end{array}$ & normal \\
\hline 06.2014 & $96 / 29,9$ & 24,5 & $122,3^{2}$ & 1092 & $\begin{array}{l}\text { Ketoconazole } \\
400 \mathrm{mg}\end{array}$ & \\
\hline 01.2015 & $91 / 28,4$ & 10,5 & $168^{2}$ & 199 & $\begin{array}{l}\text { Ketoconazole } \\
\quad 600 \mathrm{mg} \\
\text { Stereotactic } \\
\text { radiotherapy }\end{array}$ & $\begin{array}{l}\text { recurrence of } \\
\text { macroadenoma } \\
10 \mathrm{~mm} \\
\text { (fig.3) }\end{array}$ \\
\hline 12.2015 & $93 / 29$ & 11,6 & $131^{2}$ & 356 & $\begin{array}{l}\text { Ketoconazole } \\
\quad 400 \mathrm{mg}\end{array}$ & \\
\hline 03.2016 & $100 / 30,9$ & 71,9 & 214,6 & 4192 & Pasireotide & \\
\hline 05.2016 & & & & & $\begin{array}{l}\text { Ketoconazole } \\
400 \mathrm{mg}\end{array}$ & \\
\hline
\end{tabular}

During further follow-up of the patient there were periodically repeated periods of moderate subclinical hypercortisolemia, observed mainly in spring and autumn. In spring 2013, significant clinical and laboratory signs of hypercortisolemia appeared and MRI revealed pituitary macroadenoma $(15 \times 13 \times 15 \mathrm{~mm})$. In 09.2013 the patient underwent transsphenoidal surgery (atypical sparsely granulated coricotroph adenoma, ACTH (+), MIB1>20\%). Postoperative MRI didn't show tumor remnant, but moderate hypercortisolemia was present. Because of increased levels of cortisol and ACTH, the patient restarted ketoconazole treatment (from 04.2014). A follow-up MRI (01.2015) demonstrated recurrence of the tumor. Patient was disqualified from a second surgery, in 04.2015 stereotactic radiotherapy was performed (Cyber Knife, total dose 20Gy/tumor). Since November 2015 till April 2016 he received pasireotide, but no response was observed. Currently the patient is being treated with ketoconazol $(400 \mathrm{mg} /$ day) because of persistent hypercortisolemia. Other therapeutic options for the future are currently being considered (Themozolomide, adrenalectomy).

\section{Conclusions.}

The authors present long-term difficulties in establishing the diagnosis of Cushing's disease, mainly because of cyclic nature, long period of remission and diagnostic limitations in finding the source of ACTH excess. Delay in diagnosis of Cushing's disease leads to worse effects of treatment and the need to search for new therapeutic approaches.

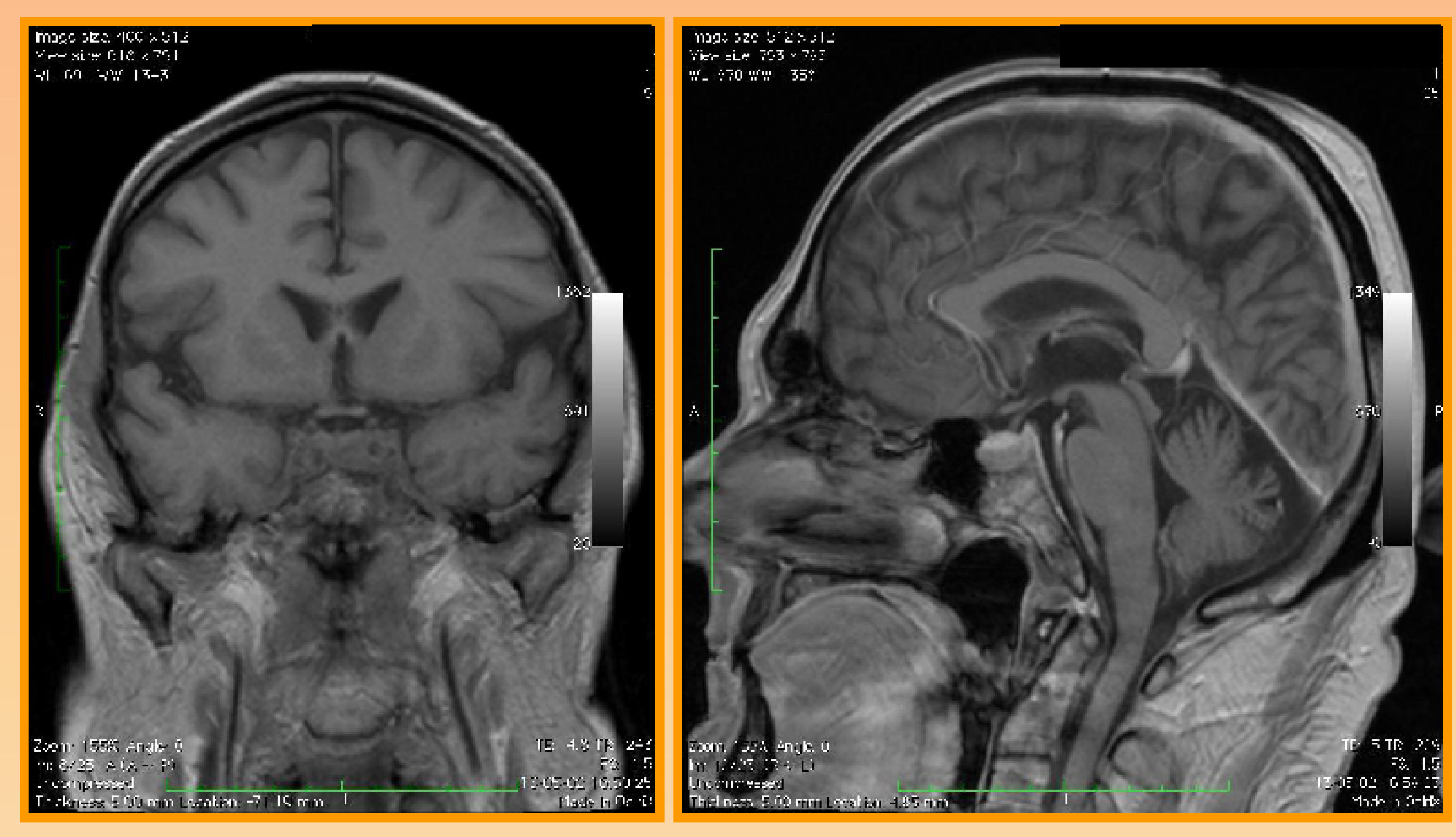

Fig. 1 and 2. MRI picture of corticotroph macroadenoma in patient with Cushing's disease (05.2013)

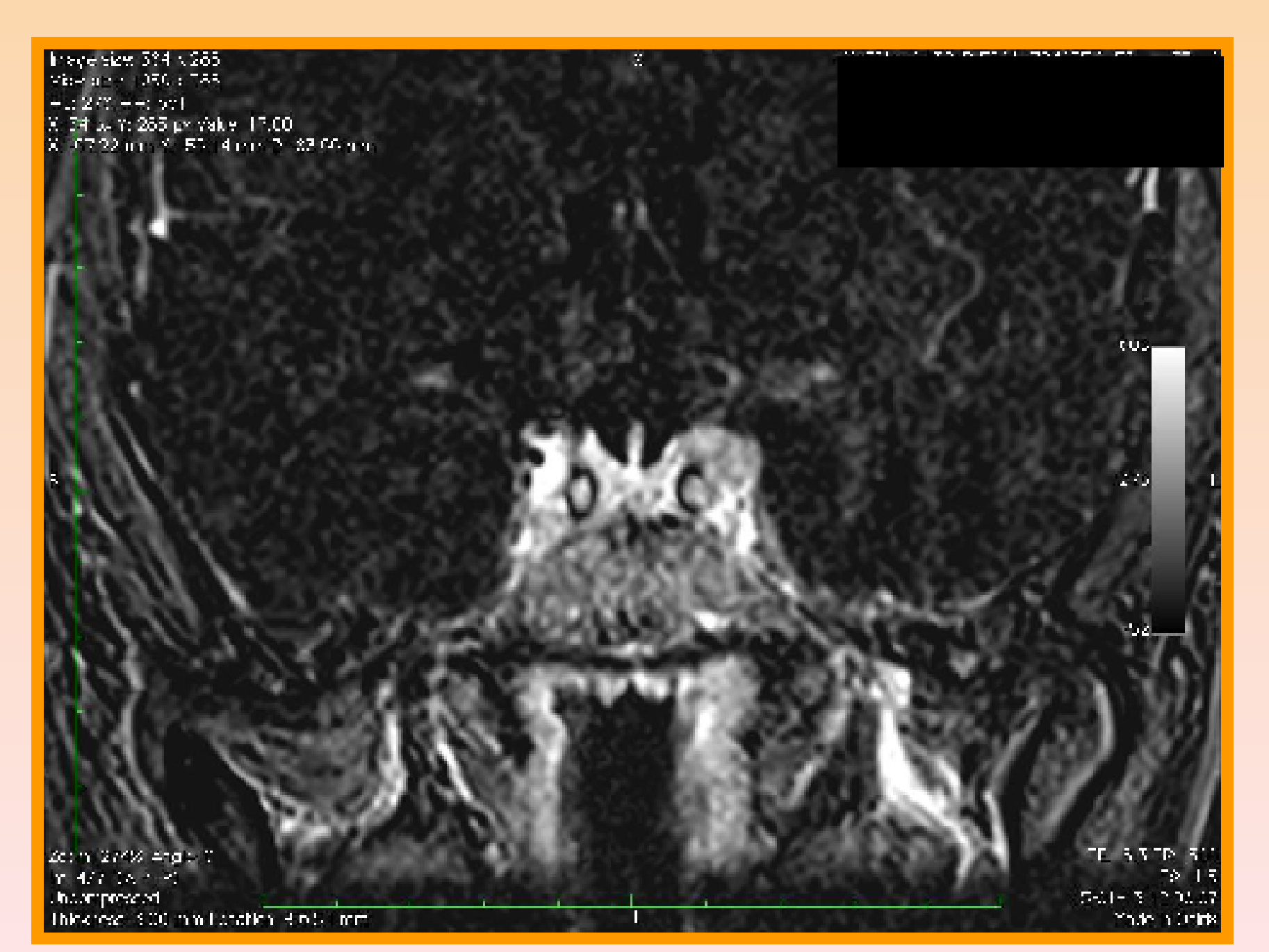

Fig. 3.

MRI picture of recurrent corticotroph macroadenoma in patient with Cushing's disease (01.2015) 